\title{
Kinetics of Myristic Acid Following Accidentally Induced Septic Response
}

\author{
Roman Zazula ${ }^{1}$, Miroslav Průcha ${ }^{2}$, František Pehal ${ }^{2}$, Kseniya Dryahina ${ }^{3}$, \\ Michal Moravec ${ }^{1,4}$, Martin Müller', Tomáš Nejtek ${ }^{1,4}$ \\ ${ }^{1}$ Department of Anesthesiology and Intensive Care, First Faculty of Medicine, \\ Charles University and Thomayer Hospital, Prague, Czech Republic; \\ ${ }^{2}$ Department of Clinical Biochemistry, Hematology and Immunology, Na Homolce \\ Hospital, Prague, Czech Republic; \\ ${ }^{3}$ Department of Chemistry of lons in Gaseous Phase, J. Heyrovský Institute \\ of Physical Chemistry, Academy of Sciences of the Czech Republic, Prague, \\ Czech Republic; \\ ${ }^{4}$ Faculty of Military Health Sciences, University of Defence, Hradec Králové, \\ Czech Republic
}

Received July 4, 2019; Accepted September 4, 2019.

Key words: Biomarkers - Sepsis - Myristic acid - Kinetics

\begin{abstract}
Myristic acid was identified as a metabolite with the highest diagnostic sensitivity and specificity in the metabolome of patients with bacteraemia.

Subsequently, its significant decrease was observed in patients in septic shock not responding to treatment. In our study we have captured myristic acid serum level kinetics in 96 hours following accidental intravenous self-administration of eubiotic Hylak forte causing infection-like systemic inflammatory response syndrome (SIRS). To our knowledge, this is the first time the kinetics of myristic acid levels is presented in a septic patient. Myristic acid was evaluated in comparison with other inflammatory biomarkers and with its level in a control group of healthy subjects. Myristic acid levels during septic response were significantly elevated in comparison with the control group. The peak level was recorded almost immediately after the insult with a gradual decrease within 96 hours. Myristic acid appears to be a promising biomarker in sepsis diagnostics, further research by our group into this topic is ongoing.
\end{abstract}

This study was supported by Ministry of Health of the Czech Republic - DRO (Thomayer Hospital - TN 00064190).

Mailing Address: Assoc. Prof. Roman Zazula, MD., PhD., Department of Anesthesiology and Intensive Care, First Faculty of Medicine, Charles University and Thomayer Hospital, Vídeňská 800, 14059 Prague 4, Czech Republic; Phone: +420 261083 811; Fax: +420 261083 393; e-mail: roman.zazula@ftn.cz 


\section{Introduction}

Although clinical features are still the cornerstone in diagnosis of sepsis, there are various biomarkers which can help physicians to confirm or question the diagnosis (Prucha et al., 2015). It has been 20 years since Brunkhorst et al. (1998) described the kinetics of procalcitonin (PCT) levels in iatrogenic sepsis. Study of Kauppi et al. (2016) identified myristic acid as a metabolite with the highest sensitivity of 1.00 (95\% Cl 0.85-1.00) ( Cl - confidence interval) and specificity of 0.95 (95\% $\mathrm{Cl}$ 0.74-0.99) in the metabolome of septic patients with bacteraemia. Cambiaghi et al. (2017) observed its significant decrease in nonresponders to the treatment of septic shock. We present a rare case of sepsis-like inflammatory response to accidental intravenous administration of the Hylak forte eubiotic preparation (germfree concentrate of metabolites of Escherichia coli, Enterococcus faecalis, Lactobacillus acidophils and Lactobacillus helvetici) with evaluation of the kinetics of several biomarkers including myristic acid. To our knowledge, this is the first time the kinetics of myristic acid serum levels following septic insult are being described.

\section{Case report}

A 30-year-old woman with no significant medical history was admitted to the Department of Gynaecology with suspected infection from uterine myoma necrosis. She was treated by intravenous antibiotics. On admission her laboratory tests were unremarkable except for C-reactive protein (CRP) of $300 \mathrm{mg} / \mathrm{I}$. She had stable vital signs with no symptoms of SIRS (systemic inflammatory response syndrome). On the second day the patient accidentally self-administered $2 \mathrm{ml}$ of the Hylak forte eubiotic preparation (Merckle $\mathrm{GmbH}$ ) in her peripheral intravenous cannula. The patient developed infection-like systemic inflammatory response syndrome shortly afterwards, she became febrile $\left(39.6^{\circ} \mathrm{C}\right)$ with tachycardia $(125$ beats $/ \mathrm{min})$ and tachypnoea (22 breaths/min), her blood pressure remained normal. The treatment consisted of fluid resuscitation and corticosteroids, intravenous antibiotic was continued. Remission of the clinical symptoms occurred within 12 hours after the event. The patient had no signs of organ dysfunction and was discharged on day 5. During a period of 96 hours following the accidental injection of Hylak, serum levels of CRP, PCT, interleukin 6 (IL-6), presepsin, copeptin and free myristic acid were evaluated. The myristic acid levels were determined by the gas chromatography/ mass spectrometry method. Gas chromatograph with flame ionisation detector was used to eliminate quantification errors of mass spectrometry scanning. Very shortly after the insult, we recorded the peak level of myristic acid reaching 10 -fold higher value $(230.9 \mu \mathrm{mol} / \mathrm{I})$ than the reference range, with a gradual decrease in the 96 hours to $26.6 \mu \mathrm{mol} / \mathrm{I}$. The reference values were determined as the median of myristic acid levels measured in healthy subjects (18.9 $\mu \mathrm{mol} / \mathrm{l}$; $\min 7.8$; $\max 27.7$; $n=66)$. Myristic acid was not detected in the eubiotic itself. All the biomarkers under evaluation showed dynamics typical of septic inflammatory response. CRP levels, significantly elevated at the time of admission due to the underlying disease and 


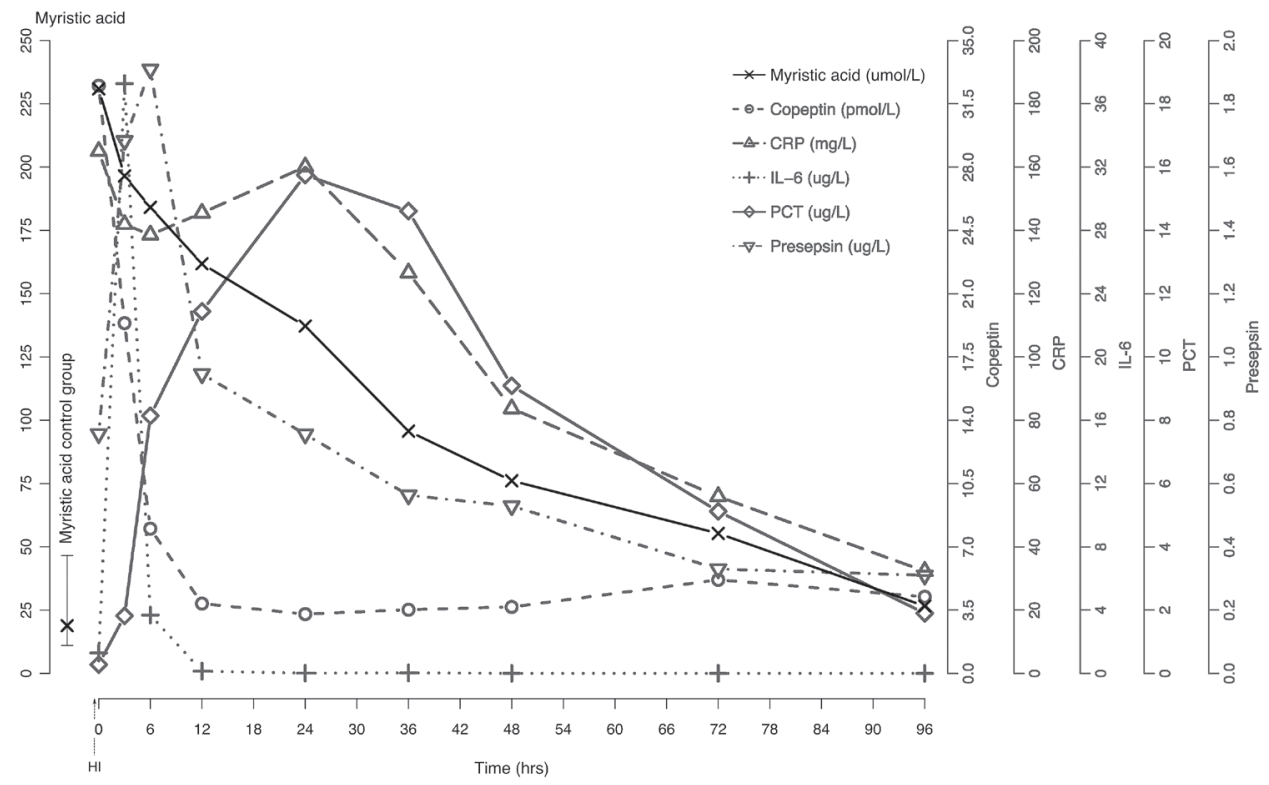

Figure 1 - Myristic acid serum levels compared to serum levels of copeptin, C-reactive protein, interleukin-6, procalcitonin and presepsin during a period 96 hours following accidental injection of Hylak forte $(\mathrm{HI}-$ Hylak forte injection 23 minutes before time TO. Reference range in myristic acid control group is presented as median (minimum; maximum).

monitored following the Hylak injection, exhibited a second mild peak 24 hours after the insult (Figure 1).

\section{Discussion}

Of interest is the ability of germless concentrate administered intravenously to trigger SIRS with a significant elevation of selected septic biomarkers. While the preparation may be sterile, it undoubtedly contains pathogen-associated molecular patterns (PAMPs) that can evoke a systemic response, and it is well known that infusion of these (for example lipopolysaccharide) can evoke a similar response to sepsis. Arguably, the clinical course was not as severe as one would expect after an intravenous application of live bacteria. The kinetics measurements of selected biomarkers of sepsis and myristic acid after accidentally induced iatrogenic sepsis were inspired by the study of Kauppi et al. (2016), who identified six metabolites in blood samples associated with the diagnosis of sepsis with bacteraemia. Myristic acid stands out of those six as the metabolite with the highest sensitivity and specificity in this metabolomic study. The biomarker alone outperformed even the traditional combination of laboratory findings and SIRS criteria recommended for sepsis diagnosis. The interesting question remains as to the origin and the role of this monosaturated organic acid of linear structure with the already described 
atherogenic and thrombogenic potential (Zong et al., 2016). Myristic acid chains are incorporated in lipid $A$ in different quantities in various species of Gram-negative bacteria (Steimle et al., 2016). However, in the same laboratory using the identical method, myristic acid was not detected in the eubiotic Hylak forte. Myristic acid is also a component of cellular membranes, it can be covalently linked to proteins and serve to anchor signal proteins by insertion of the acyl chain into the lipid bilayer (Jennings and Linder, 2009; Stillwell, 2016). Protein N-myristoylation has been shown to be an important evolutionarily conserved modification of proteins implicated in different physiological processes like cell proliferation, differentiation, survival, and cell death (Udenwobele et al., 2017). It is possible, that alterations in lipid metabolism linked to changes in cellular energy production during inflammatory response activation may be responsible for elevated levels of myristic acid in early stages of a septic episode (Kauppi et al., 2016; Cambiaghi et al., 2017).

\section{Conclusion}

Myristic acid shows a potential to be a promising marker for early identification of septic patients. The clinical relevance of these findings is currently systematically evaluated, focusing on what new information a marker can provide in describing patients with systemic infections (ClinicalTrials.gov NCT03314831).

\section{References}

Brunkhorst, F. M., Heinz, U., Forycki, Z. F. (1998) Kinetics of procalcitonin in iatrogenic sepsis. Intensive Care Med. 24, 888-889.

Cambiaghi, A., Pinto, B. B., Brunelli, L., Falcetta, F., Aletti, F., Bendjelid, K., Pastorelli, R., Ferrario, M. (2017) Characterization of a metabolomic profile associated with responsiveness to therapy in the acute phase of septic shock. Sci. Rep. 7, 9748.

Jennings, B. C., Linder, M. E. (2009) Regulation of G proteins by covalent modification. In: Handbook of Cell Signaling, $2^{\text {nd }}$ Edition. Bradshaw, R., Dennis, E., Pp. 1629-1634, Academic Press, Cambridge.

Kauppi, A. M., Edin, A., Ziegler, I., Mölling, P., Sjöstedt, A., Gylfe, Å., Strålin, K., Johansson, A. (2016) Metabolites in blood for prediction of bacteremic sepsis in the emergency room. PloS One 11, e0147670.

Prucha, M., Bellingan, G., Zazula, R. (2015) Sepsis biomarkers. Clin. Chim. Acta 440, 97-103.

Steimle, A., Autenrieth, I. B., Frick, J. S. (2016) Structure and function: Lipid A modifications in commensals and pathogens. Int. J. Med. Microbiol. 306, 290-301.

Stillwell, W. (2016) An Introduction to Biological Membranes: Composition, Structure and Function. Elsevier Science, London.

Udenwobele, D. I., Su, R. C., Good, S. V., Ball, T. B., Varma Shrivastav, S., Shrivastav, A. (2017) Myristoylation: An important protein modification in the immune response. Front. Immunol. 8, 751.

Zong, G., Li, Y., Wanders, A. J., Alssema, M., Zock, P. L., Willett, W. C., Hu, F. B., Sun, Q. (2016) Intake of individual saturated fatty acids and risk of coronary heart disease in US men and women: two prospective longitudinal cohort studies. BMJ 355, i5796. 